\title{
Partager sa connaissance du système lexical pour développer ses savoirs lexicaux en fin d'école primaire (9-11 ans)
}

\author{
Anne Sardier \\ ESPE université Clermont-Auvergne, ACTé EA4281 \\ anne.sardier@uca.fr
}

\begin{abstract}
Résumé. La présente contribution s'inscrit dans une réflexion sur l'enseignementapprentissage du lexique à l'école primaire. Elle propose d'interroger les effets des échanges entre pairs sur les apprentissages lexicaux d'élèves de fin de cycle 3 (9-11 ans). Des temps de discussions sont proposés dans huit classes de fin d'école primaire. Ces temps permettent aux élèves de mobiliser notamment les différentes structures soustendant le système lexical. Afin de pouvoir en évaluer les effets, le protocole de recherche se déploie sous la forme d'un dispositif comparatif à partir de la variable discussion. Nous rendons compte des premiers résultats de cette recherche encore en cours. Les données constituées notamment de productions d'élèves sont analysées à partir de l'indicateur principal de réussite qui est le réemploi lexical des unités étudiées en classe. Les premiers résultats tendent à montrer que le fait de prévoir, dans les séances consacrées au lexique, des temps de discussion mobilisant les différentes structures du système au lexique favorise le réemploi lexical.
\end{abstract}

\begin{abstract}
Share the knowledge of the lexical system to develop some lexical knowledge at the end of primary school. This contribution is part of a reflection on the teaching-learning of lexicon in primary school. It proposes to question the effects of exchanges between peers on the lexical learnings of students at the end of cycle 3 (9-11 years). Discussion times are offered in eight end-of-primary classes. These times allow students to mobilize in particular the different structures underlying the lexical system. In order to evaluate the effects of this discussions, the search protocol deploys comparative device from the discussion variable. We report the first results of this research still in progress. Data consisting mainly of pupils productions are analyzed using the lexical reuse of the units studied in class as the main indicator of success. The first results tend to show that the fact of foreseeing, in the sessions devoted to the lexicon, times of discussion mobilizing the different structures of the lexicon system favors lexical re-use.
\end{abstract}

\begin{abstract}
Notre proposition s'appuie sur une recherche en cours dans le département du Cantal (Auvergne, France) impliquant huit classes de cycle 3 (9-11 ans). Considérant que le rôle des échanges entre pairs est assez bien étudié concernant différents apprentissages linguistiques, mais qu'il reste fort peu exploré concernant les apprentissages lexicaux, nous souhaitons présentement interroger le rôle de ces échanges sur le développement des apprentissages lexicaux d'élèves de fin d'école primaire. La classe formant une « communauté d'apprentissages » (Laferrière, 2005, p.9), nous pensons que les élèves d'une même classe, grâce à leurs échanges et à leur vécu commun, peuvent parvenir à des consensus quant au sens et à l'emploi des unités lexicales qu'ils utilisent et qu'ils forment ainsi une communauté d'ordre lexical restreinte à l'échelle de la classe. Par ailleurs, étant donné que des recherches récentes tendent à montrer
\end{abstract}


qu'en didactique du lexique il est déterminant de considérer les différentes structures sous-tendant le système lexical (Tremblay \& Polguère, 2014 ; Sardier, 2015), nous pensons qu'il est nécessaire que les situations d'échange permettent de mobiliser les structures sémantiques, morphologiques et syntagmatiques. Nous faisons dès lors l'hypothèse que la constitution de cette communauté d'apprentissages lexicaux, établie notamment à travers les échanges entre pairs, peut favoriser le développement de la compétence lexicale. Les discussions engagées, en incitant les élèves à mobiliser les différentes structures du système lexical, devraient ainsi favoriser les apprentissages lexicaux. Afin de vérifier cette hypothèse, nous proposons d'engager les élèves dans des situations d'enseignementapprentissage dans lesquelles ils sont invités à prendre le temps de discuter des unités lexicales qu'ils étudient. Le protocole déployé nous a d'abord amenée à sélectionner les unités lexicales à étudier dans les classes. Puis, dans cette recherche de type expérimental, nous avons différencié deux groupes au vu de la variable discussion: les classes du groupe A bénéficient des discussions, contrairement aux classes du groupe B. Les données analysées sont constituées d'un prétest écrit permettant un diagnostic sur l'emploi des unités lexicales étudiées en classe, des notes prises lors des échanges et d'un posttest écrit évaluant le réemploi des unités lexicales. Cette répartition des classes et le recueil des données ainsi établi pourraient permettre de savoir si la communauté constituée dans le groupe A grâce aux discussions sur le lexique a favorisé ces élèves par rapport à ceux du groupe B. Dans cette recherche, nous avons pris le parti de ne pas nous focaliser sur l'analyse des interactions, mais plutôt sur l'analyse des productions écrites des élèves, au vu de la mise en œuvre ou non des discussions en classe. Nous interrogeons dès lors dans un premier temps le concept de discussion pour en proposer notre définition au regard des apprentissages lexicaux ; puis nous présentons le dispositif de recherche mis en place dans les sept classes de cycle 3 ; enfin, nous analysons les résultats obtenus à l'issue de la mise en œuvre du dispositif.

\section{Cadre théorique}

\subsection{La didactique du lexique : un enseignement-apprentissage organisé}

Différents facteurs sont à considérer en didactique : l'organisation du lexique, l'organisation des séances de classe et l'organisation des interactions.

Depuis plusieurs années, les recherches en didactique du lexique prônent un enseignement organisé autour des différentes structures du lexique (par exemple: Chanfrault-Duchet, 2004 ; Polguère \& Tremblay, 2014 ; Sardier, 2016). Les propositions en didactique du lexique doivent alors considérer les axes sémantique, syntagmatique et morphologique qui sous-tendent l'organisation du système lexical. Les livrets-outils que nous proposerons aux élèves s'efforcent ainsi d'engager une réflexion selon ces trois axes, avec une priorité accordée aux axes sémantique et syntagmatique puisque notre travail s'articule surtout autour du sens des unités lexicales étudiées avec les élèves. Lors des interactions, le sens sera discuté au sein de la classe afin d'aboutir à un consensus sur les acceptions des unités étudiées. Mais l'axe morphologique n'est pas totalement oublié puisque les élèves sont appelés à proposer des unités lexicales de même famille.

Par ailleurs, les séances consacrées au lexique doivent être proposées selon une certaine progression. Cette progression concerne à la fois les notions lexicales (Polguère \& Tremblay, 2014) et la prise en compte du processus de mémorisation du lexique (Sardier, 2015). Il s'agit alors de considérer l'organisation mémorielle des réseaux lexicaux qui est mouvante et réorganisée constamment en fonction des nouvelles acquisitions. Notons aussi que le processus menant au réemploi lexical est long et que le facteur temps est ici à considérer (Sardier \& Grossmann, 2010). Le dispositif que nous proposons débute par une séance qui consiste à faire émerger le lexique disponible des élèves afin que les unités lexicales nouvellement étudiées puissent venir s'y agréger. Ce lexique disponible pour chacun est partagé au sein de la classe via les interactions verbales prévues et organisées dans les séances consacrées au lexique.

En outre, nous souhaitons prendre également en compte «certaines pratiques d'oral [qui] peuvent enclencher l'évolution des élèves et outiller la construction des savoirs » (Jaubert \& Rebière, 2002, p. 163). Ainsi, les échanges ont lieu à des moments précis dans l'organisation du diapositif didactique : en début de parcours pour constituer une base lexicale partagée, en fin de parcours pour partager les 
avancées par rapport à cette première base partagée. Le rôle de l'enseignant consiste alors à étayer les échanges pour que d'une part les élèves aboutissent à un premier consensus et pour que d'autre part ils élargissent leur champ lexical par échanges quant aux avancées de chacun. De la sorte, les interactions verbales participent de la réflexivité en permettant d'abord une "prise de distance à l'égard de l'expérience immédiate» (Chabanne \& Bucheton, 2002, p. 5), en proposant des activités métalinguistiques et en créant un « espace intersubjectif commun » (Chabanne \& Bucheton, 2002, p. 7).

\subsection{Les interactions verbales en didactique du lexique : essai de définition}

Le cadre communicatif de l'interaction verbale est pour nous circonscrit à la classe. Ainsi, en nous appuyant sur les réflexions de Kerbrat-Orecchioni ([1990] 1998), nous pouvons dire que le cadre spatiotemporel sera celui de la séance de vocabulaire dispensée dans la salle de classe. Or, il nous semble que ce cadre prédétermine le but des interactions alors engagées : il s'agit pour l'enseignant de mener ses élèves vers des discussions relatives au lexique et pour les élèves, sachant qu'ils se situent en séance de vocabulaire, il s'agit de s'engager dans ses discussions préorientées par le thème de la séance. L'enseignant propose à ses élèves des « interactions finalisées » (Kerbrat-Orecchioni, [1990] 1998, p. 80) et tendues vers un but déterminé par les apprentissages lexicaux. Dans le cadre didactique qui est le nôtre, les participants sont dès lors envisagés comme représentant un «ensemble de savoirs » (KerbratOrecchioni, [1990] 1998, p. 82) lexicaux. Ces savoirs lexicaux sont convoqués au fur et à mesure des temps d'interactions organisés par l'enseignant et constituent la connaissance partagée par les intervenants. Dans le cadre des interactions verbales organisées dans les séances consacrées à l'étude du lexique, les élèves sont ainsi invités à mobiliser leurs connaissances déjà existantes en se basant sur l'organisation du système lexical pour nourrir leur réflexion à travers leurs échanges oraux.

De ce fait, en discutant des unités lexicales étudiées, les élèves partagent, expliquent et justifient leurs propres acceptions de ces unités. Avec l'aide de leur enseignant, ils peuvent, à l'issue de ces échanges, aboutir à des consensus quant aux sens des unités étudiées. En outre, dans la mesure où ces oraux réflexifs ${ }^{\mathrm{i}}$ ayant pour but la construction d'une compétence comportent « une composante argumentative importante » (Kerbrat-Orecchioni, [1990] 1998, p. 118), nous pouvons également préciser, à l'instar de Kerbrat-Orecchioni, qu'il s'agit de discussions. Étant donné que les échanges que nous évoquons se construisent autour du lexique, nous pouvons dire que l'enseignant organise alors des discussions lexicales que nous supposons favorables au développement des apprentissages lexicaux. Nous pensons en effet que le fait de mettre en œuvre des interactions verbales prévues et organisées va pouvoir favoriser le développement de la compétence lexicale des élèves.

Afin d'organiser judicieusement ces échanges pour permettre une co-construction de la pensée (Chabanne \& Bucheton, 2002), nous proposons de poser aux élèves un problème lexical qui pourra être discuté oralement en commun. Ainsi l'enseignant s'appuie sur les échanges entre pairs pour résoudre un problème qui s'est posé, ou plutôt qu'il a posé aux élèves. Or, dans les activités scolaires portant sur le lexique, la discussion lexicale est assez rarement convoquée, bien qu'elle puisse avoir, comme nous souhaitons le montrer, un impact positif sur les apprentissages lexicaux.

Ainsi les interactions verbales en didactique du lexique sont pour nous des interactions finalisées : ce sont des discussions lexicales entre pairs, étayées par l'enseignant qui se base sur les connaissances préalables des élèves et les structures sémantiques, syntagmatiques et morphologiques du système lexical comme nous allons le préciser. Les temps d'échange que nous proposons en classe suivent ainsi ces grands principes.

\section{Méthodologie de recherche}

\subsection{Constitution des groupes participants}

Les huit classes qui participent au dispositif ont été sélectionnées en coordination avec la direction des services départementaux de l'éducation nationale du Cantal. Les classes se situent toutes dans le même bassin scolaire et ne présentent pas de grandes différences quant aux compétences des élèves. Elles se 
différencient essentiellement par le nombre d'élèves présents dans les différents niveaux qui les composent. Ainsi certaines sont des classes rurales uniques regroupant l'ensemble des élèves de la maternelle au primaire et, à ce titre, regroupent peu d'élèves dans chaque niveau. D'autres sont des classes à double niveaux ou à simple niveaux et présentent un plus grand nombre d'élève dans chacun des niveaux qui les composent. Deux groupes ont ainsi pu être constitués afin de pouvoir évaluer l'impact des interactions verbales sur les apprentissages :

- Un groupe de classes (groupe A) dans lesquelles les élèves bénéficient des échanges verbaux soit par l'intermédiaire du réseau à distance quand les élèves de ces classes étaient trop peu nombreux pour discuter (compte tenu de la topographie des classes sélectionnées, nous avons alors présupposé que les classes comportant moins de huit élèves par niveau présentaient trop peu d'élèves pour engager des discussions lexicales telles que nous les avons définies précédemment), soit en présence quand les élèves étaient suffisamment nombreux pour engager des discussions lexicales (classe de plus de huit élèves)

- Un groupe de classes (groupe B) dans lesquelles les élèves ne peuvent bénéficier de l'instauration de ces discussions car ils sont trop peu nombreux pour susciter des échanges productifs constitutifs des discussions lexicales (moins de huit élèves par niveau).

Cette répartition amène deux remarques d'importance :

- D'une part elle permet d'établir une comparaison entre le groupe test et le groupe témoin, tout en considérant que le groupe B pourra connaitre cependant des épisodes d'échanges mais qui, nous le verrons, sont moins développés que ceux ayant lieu dans le groupe $\mathrm{A}$

- D'autre part, bien que nous nous questionnions présentement sur l'efficience de la communauté lexicale via les interactions verbales, elle invite également à interroger l'impact des échanges en réseau à distance sur les apprentissages lexicaux des élèves peu nombreux en classe, ce à quoi nous nous employons par ailleurs ii

Afin de construire les moyens d'une comparaison entre les classes, l'ensemble des élèves travaillent sur le même corpus d'unités lexicales et suivent le même enseignement comme nous allons le voir ci-dessous.

\subsection{Un choix : le lexique verbal}

Tous les élèves de l'ensemble des classes travaillent sur le même corpus d'unités lexicales. Plusieurs critères ont guidé notre sélection d'unités à travailler.

Premièrement, les verbes, bien qu'étant le pivot de la phrase française, sont souvent moins étudiés que les noms ou les adjectifs. Dans le souci de proposer une didactique du lexique prenant en compte l'étude des verbes, c'est sur cette classe grammaticale que nous nous sommes focalisée.

Deuxièmement, il s'agissait pour nous de proposer un corpus d'unités pouvant être réemployées dans les productions écrites ou orales dans toutes les classes, mais indépendamment des progressions des enseignants. Il nous paraissait en effet difficile d'imposer à tous des progressions identiques au service du réemploi lexical. Nous avons alors sélectionné des verbes de parole car il nous a semblé que ces verbes pouvaient être mobilisés dans des situations variées de lecture ou d'écriture.

Troisièmement, l'un des objectifs de l'enseignement du lexique est l'accroissement du stock lexical des élèves. Nous avons donc consulté la liste de fréquence afin de ne pas proposer des verbes trop fréquents ou trop familiers tels dire ou même répondre, que les élèves connaissent déjà bien au cycle 3 , mais plutôt des verbes qu'ils ont pu rencontrer sans pour autant qu'ils leur soient familiers. Dire étant un terme générique très fréquemment employé par les élèves, comme nous l'ont fait remarquer les enseignants, nous avons cherché à outiller les élèves afin de leur permettre d'exprimer des nuances dans les manières de dire pour qu'ils puissent mieux comprendre ou rendre compte des intentions des personnages, ou autres individus proférant des paroles rapportées.

Enfin, l'analyse sémantique pouvant favoriser une organisation méthodique de l'enseignement du lexique (Aurnague \& Garcia-Debanc, 2016), nous avons voulu structurer le champ des verbes de parole pour outiller au mieux les élèves. En considérant que les liens entre lexique et syntaxe sont difficilement 
dissociables, nous nous sommes appuyée sur l'analyse proposée par Lamiroy \& Charolles qui traite de l'(in)transitivité des verbes de parole. Ces verbes sont analysés sémantiquement selon :

« •(1) la source des paroles: verbes comme réciproquer ou commenter qui impliquent qu'il y ait eu des paroles antérieures ;

- (2) la forme du message: verbes comme zézayer ou murmurer qui indiquent la façon dont les paroles sont proférées ;

- (3) le caractère non programmé du message: verbes tels que papoter ou bavarder qui indiquent que le contenu des paroles n'est pas programmé au préalable ;

- (4) le locuteur: verbes qui spécifient soit l'état mental du locuteur (ex. délirer) soit une attitude psychologique (ex. fulminer) ;

- (5) l'acte de langage: verbes dénotant différents type d'actes de langage tels qu'ils ont été établis par Searle (1979/82) tels que décréter (acte déclaratif), (dé)conseiller (acte directif), implorer (acte assertif) et féliciter (acte expressif) ;

- (6) le caractère générique du verbe: verbes neutres tels que parler ou dire » (Lamiroy \&

Charolles, 2008, p. 3 et 4).

En synthétisant les propositions de Lamiroy \& Charolles dans une perspective liée à la didactique du lexique, nous pouvons avancer que les verbes de parole entrent dans deux catégories : les items référencés par les auteurs en (1) et (3) réfèrent prioritairement au déroulement de l'échange (par exemple réciproquer implique l'existence d'un échange antérieur et papoter implique l'existence d'un échange réciproque), alors que les items référencés en (2), (4) et (5) réfèrent prioritairement à l'expression d'une émotion ressentie par le locuteur (par exemple murmurer est fréquemment lié à la peur d'être entendu, peur pouvant avoir des origines diverses). Ainsi, nous avons établi une liste qui propose des verbes sélectionnés en fonction des deux sèmes présents dans les verbes de paroles :

- prononcer des sons souvent en lien avec une émotion (a)

- participer à un échange discursif (b).

Comme indiqué précédemment, cette sélection, établie en accord avec les enseignants des classes participantes, s'inspire des listes de fréquence (en excluant les verbes les plus fréquents rencontrés déjà par les élèves et dont nous présupposons qu'ils sont connus), et elle retient des verbes supposés moins connus. Pour chaque verbe de parole, les deux sèmes restent présents, mais l'un domine par rapport à l'autre. En fonction de ces dominantes sémiques, il est ainsi possible de proposer un classement des verbes sélectionnés avec les enseignants :

- (a) = force de la voix souvent liée à une dimension émotionnelle : hurler, murmurer, marmonner,

- $\quad$ (b) = indication d'une étape du dialogue entre les personnages-locuteurs participant à l'échange : conclure, déclarer, répliquer.

Nous tenons compte alors du fait que «l'expression d'un procès, [...], n'est pas attribuable au seul verbe mais à l'ensemble des unités en présence » (Triquenot, 2011, p. 165). Ainsi, afin d'affiner l'analyse et de mettre en exergue les sèmes dominants pour que les élèves les perçoivent mieux, il est alors possible de mobiliser le concept de cotexte ${ }^{\text {iii }}$ afin de préciser le sens des verbes. Le cotexte tel que nous l'envisageons est alors représenté par les cooccurrents de sens plein fréquemment employés avec les unités étudiées. Cette définition du cotexte permet de proposer aux élèves des unités lexicales qui peuvent se combiner avec les verbes étudiés afin de mieux les comprendre. Ainsi, l'analyse peut se résumer sous la forme d'un tableau synthétique. Dans un but didactique nous intégrons à la liste des verbes à étudier des éléments cotextuels pouvant favoriser la compréhension des expressions ainsi construites. Ces éléments seront mobilisés durant les séances consacrées au lexique comme nous le verrons plus loin.

Tableau 1. Synthèse de l'analyse sémantique des verbes de parole à des fins didactiques

\begin{tabular}{|l|l|l|}
\hline \multicolumn{2}{|l|}{ Verbes de parole étudiés } & Eléments cotextuels fréquents \\
\hline Force de la voix et & Hurler & de peur, de colère... \\
\hline
\end{tabular}




\begin{tabular}{|l|l|l|}
\hline \multirow{2}{*}{ dimension émotionnelle } & Murmurer & discrètement / à part... \\
\cline { 2 - 3 } & Marmonner & $\begin{array}{l}\text { entre ses dents... } \\
\text { tout bas } / \text { sourdement... }\end{array}$ \\
\hline \hline \multirow{2}{*}{$\begin{array}{l}\text { Etape du dialogue entre les } \\
\text { personnages }\end{array}$} & Conclure & $\begin{array}{l}\text { la conversation... } \\
\text { sèchement } / \text { rapidement... }\end{array}$ \\
\cline { 2 - 3 } & Déclarer & $\begin{array}{l}\text { sa satisfaction... } \\
\text { formellement... }\end{array}$ \\
\cline { 2 - 3 } & Répliquer & $\begin{array}{l}\text { aux attaques... } \\
\text { durement... }\end{array}$ \\
\hline
\end{tabular}

Ce type de sélection et de classement d'un corpus d'unités lexicales peut favoriser la précision du sens par les élèves et permettre ainsi de répondre également en partie au deuxième objectif de l'enseignement du lexique qui consiste à engager les élèves dans une réflexion relative au fonctionnement du système lexical. Cette sélection permet en effet de mettre en regard différentes unités en analysant finement leur champ sémantique comme nous allons le voir dans les activités proposées aux élèves.

\subsection{Intégration des interactions verbales en didactique du lexique}

Nous proposons deux séances en classe pour étudier ces six verbes de parole.

Dans un premier temps nous cherchons à faire émerger le lexique disponible chez les élèves en discutant des verbes qu'ils connaissent déjà et en en faisant émerger les sens connus. Grâce aux discussions, ce premier temps permet d'aboutir à un consensus transitoire relatif au sens des verbes, consensus qui sera complété par la suite. Dans un deuxième temps nous proposons un problème lexical par l'intermédiaire de phrases dans lesquelles les verbes prennent des acceptions différentes de celles évoquées précédemment. Ce deuxième temps permet ainsi de préciser les sens de verbes en mobilisant notamment la dimension syntagmatique du lexique et en comparant les verbes entre eux. Les élèves sont ensuite invités à manipuler les verbes dans un jeu d'écriture. Dans un troisième temps les élèves partagent leurs propositions écrites et justifient leurs choix. Ce troisième temps permet ainsi de produire un consensus plus abouti pour chacun des verbes étudiés. Enfin, les élèves doivent s'entrainer à réemployer des verbes dans une production écrite. Ainsi divers types d'activités mobilisant les différentes structures du système et se déroulant à l'oral et à l'écrit sont mises en œuvre pour favoriser la mémorisation des unités lexicales étudiées.

Deux livrets intitulés respectivement Se questionner sur six verbes de parole et Connaitre et utiliser six verbes de parole constituent les supports des séances de classe. L'ensemble des classes suivent le même déroulement, mais les interactions verbales sont grandement réduites dans le groupe des classes ne comportant que deux à huit élèves par niveau. Le tableau ci-dessous synthétise le déroulement du dispositif didactique proposé :

Tableau 2. Scénario didactique comparatif intégrant les interactions verbales à l'étude du lexique

\begin{tabular}{|l|l|l|}
\hline Organisation de l'étude du lexique & Groupe A & Groupes B \\
\hline Temps 1 : Présentation des six verbes de parole & & + \\
\hline Discussion sur les sens des verbes et recherche d'un consensus & + & - \\
\hline $\begin{array}{l}\text { Temps 2 : Réactivation du lexique étudié } \\
\text { Recherche d'autres sens non évoqués en 1 (problème lexical) } \\
\text { Jeu d'écriture (production de devinettes lexicales }{ }^{\text {iv }} \text { ) }\end{array}$ & + & + \\
\hline Temps 3 : Partage des devinettes, discussion & + & + \\
& + & - \\
\hline
\end{tabular}




\begin{tabular}{|l|l|l|}
\hline Prolongement : Courte production écrite avec consigne de réemploi & + & + \\
\hline
\end{tabular}

Le tableau synthétique ci-dessus montre de quelle manière le scénario didactique permet de jouer sur la variable discussion. En effet, les discussions lexicales, sans être totalement absentes dans le groupe B, y seront largement moins développées du fait du faible nombre d'élèves.

Ce tableau fait également ressortir le caractère progressif du cheminement dans l'étude du lexique : la réorganisation du lexique des élèves peut s'effectuer grâce à une première activation de leur stock disponible, grâce à la mobilisation des structures sous-tendant le système (par exemple par recours au cotexte au moment où l'enseignant pose son problème lexical en proposant aux élèves les verbes dans un contexte phrastique où le sens est à réfléchir en fonction des éléments cotextuels, ou par le recours à la morphologie pour trouver des "mots de la même famille"), et grâce aux pratiques d'oral favorisant la réflexion en amont et la construction de champs sémantiques plus complets en aval.

\subsection{Recueil des données}

Un prétest (données écrites) permet d'évaluer à priori l'emploi des verbes de parole. Il s'agit d'une production écrite demandant aux élèves d'écrire un dialogue à partir d'une vignette de bande dessinée. Nous opérons alors un décompte du nombre d'élèves utilisant des verbes de parole dans chaque groupe. Nous analysons également la variété des verbes de parole utilisés afin de savoir quels verbes les élèves emploient déjà majoritairement et si certains de ces verbes appartiennent à notre corpus.

Le posttest (données écrites) permet d'évaluer l'emploi des verbes de parole étudiés à l'issue de la mise en œuvre. Il s'agit ici d'une production écrite demandant aux élèves d'écrire un dialogue à partir d'une situation initiale donnée. Un décompte du nombre d'élèves utilisant des verbes de parole dans chaque groupe est à nouveau réalisé. Un autre décompte permet de connaitre le nombre d'élèves utilisant les verbes étudiés dans chacun des groupes. La variété des verbes employés est également analysée. Les groupes sont comparés entre eux, de manière statistique.

Enfin, les deux livrets des élèves (données écrites) et les notes prises lors des temps de discussion (données orales) permettent une analyse comparative entre d'une part les propositions écrites et orales et d'autre part les productions finales du posttest. Notre objectif n'étant pas d'analyser les interactions, mais plutôt leurs effets sur le réemploi lexical, nous ne présentons pas pour cette raison les retranscriptions des échanges ayant eu lieu dans les classes, mais nous en extrayons les répliques qui nous semblent les plus significatives pour expliquer le réemploi au posttest.

Les séances consacrées aux verbes de parole ont eu lieu au mois de mars-avril, avant les vacances de printemps et le posttest a eu lieu après les vacances.

\section{Analyse du dispositif: impact des discussions lexicales sur les apprentissages lexicaux}

\subsection{En amont du dispositif : des différences peu marquées entre les classes}

Une première analyse des données issues du prétest nous permet de connaitre le nombre d'élèves qui utilisent déjà des verbes de parole dans leur production avant la mise en œuvre du dispositif : 


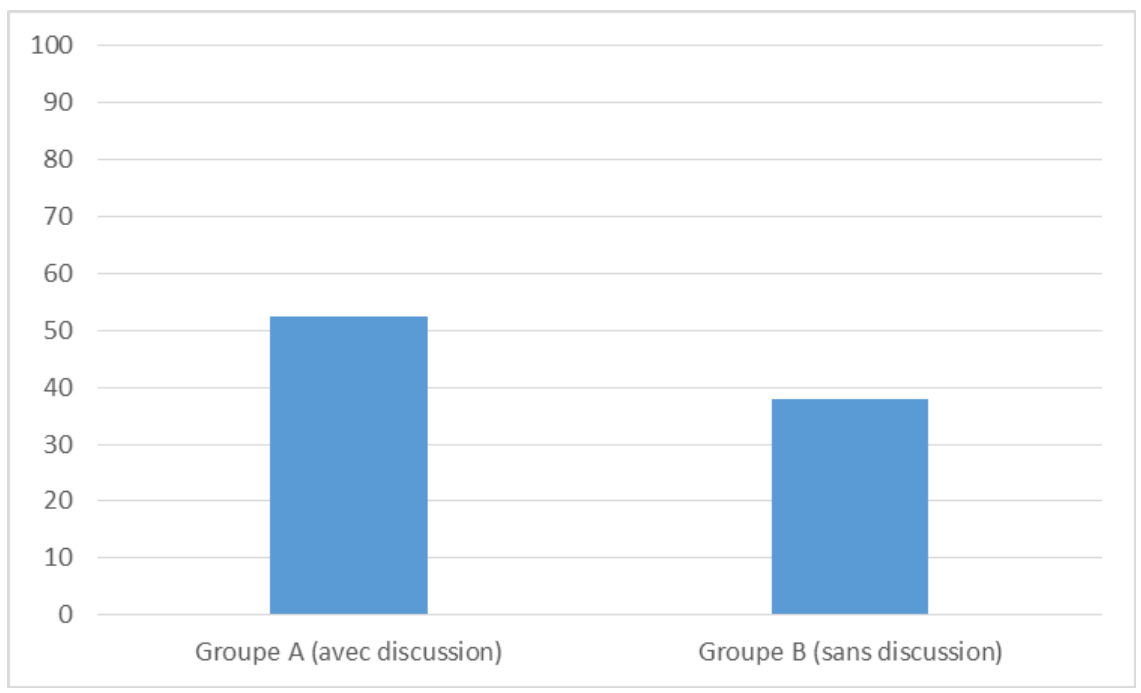

Fig. 1. Au prétest : pourcentages d'élèves utilisant des verbes de parole $(n=88)$

Le graphique ci-dessus fait penser que les élèves du groupe A utilisent intuitivement plus de verbes de parole que les élèves du groupe $\mathrm{B}$. Cependant une remarque d'importance est ici à apporter : dans une classe du groupe A, l'enseignante nous a expliqué qu'il y avait eu un échange entre elle et les élèves sur ce qu'était un dialogue avant la production écrite. Cette classe aussi la seule où tous les élèves qui utilisent au posttest des verbes de paroles recourent exclusivement au verbe dire. Cette remarque vient ainsi tempérer la différence apparente entre les deux groupes. Et nous pouvons dire qu'en amont du dispositif une assez forte proportion d'élèves utilisant des verbes de parole dans chaque groupe, malgré quelques différences. Il s'agit maintenant de savoir quels sont les verbes que les élèves emploient afin de savoir si notre corpus va effectivement permettre d'accroitre leur stock de verbes de parole.

Une deuxième analyse du prétest permet alors de connaitre quels sont les verbes employés majoritairement par les élèves en amont du dispositif :

Tableau 3. Au prétest : variété de verbes de parole utilisés en nombre d'occurrences $(n=88)$

\begin{tabular}{|l|c|c|}
\hline $\begin{array}{l}\text { Variété des verbes de } \\
\text { parole utilisés }\end{array}$ & $\begin{array}{l}\text { Groupe A } \\
\text { (avec discussion) }\end{array}$ & $\begin{array}{l}\text { Groupe B } \\
\text { (sans discussion) }\end{array}$ \\
\hline Dire & $\mathbf{2 1 8}$ & $\mathbf{1 1 5}$ \\
\hline Répondre & 7 & 0 \\
\hline Demander & 8 & 2 \\
\hline Questionner & 2 & 1 \\
\hline Crier & 0 & 1 \\
\hline Total & $\mathbf{2 3 5}$ & $\mathbf{1 1 9}$ \\
\hline
\end{tabular}

Le tableau 3 nous montre, comme nous l'attendions, que le verbe générique dire est largement suremployé par les élèves. Les autres verbes sont des verbes plus fréquents en langue et plus familiers aux élèves que ceux que nous avons sélectionnés et un seul d'entre eux, crier, réfère directement à un état émotionnel ressenti par le locuteur. Les verbes utilisés par les élèves servent ainsi plus à exprimer la chronologie de l'échange entre les personnages et que leur ressenti.

L'étude des données issues du prétest permet ainsi d'établir un état des lieux des emplois des verbes de parole par les élèves de cycle 3 et nous pouvons dire que :

$\checkmark \quad$ les élèves utilisent des verbes de parole dans l'ensemble des groupes 
$\checkmark \quad$ la variété des verbes utilisés est très limitée : le verbe dire est très majoritairement utilisé comme attendu

$\checkmark \quad$ les verbes indiquant l'émotion du locuteur sont fort peu représentés.

La question se pose maintenant de savoir si, à l'issue de l'expérience, les groupes vont présenter une différence marquée en faveur des classes dans lesquelles la communauté lexicale a pu être instaurée via les interactions verbales.

\subsection{En aval du dispositif : quels effets des discussions lexicales ?}

Nous procédons dans un premier temps, comme précédemment pour les données issues du prétest, au décompte des élèves employant des verbes de parole au posttest :

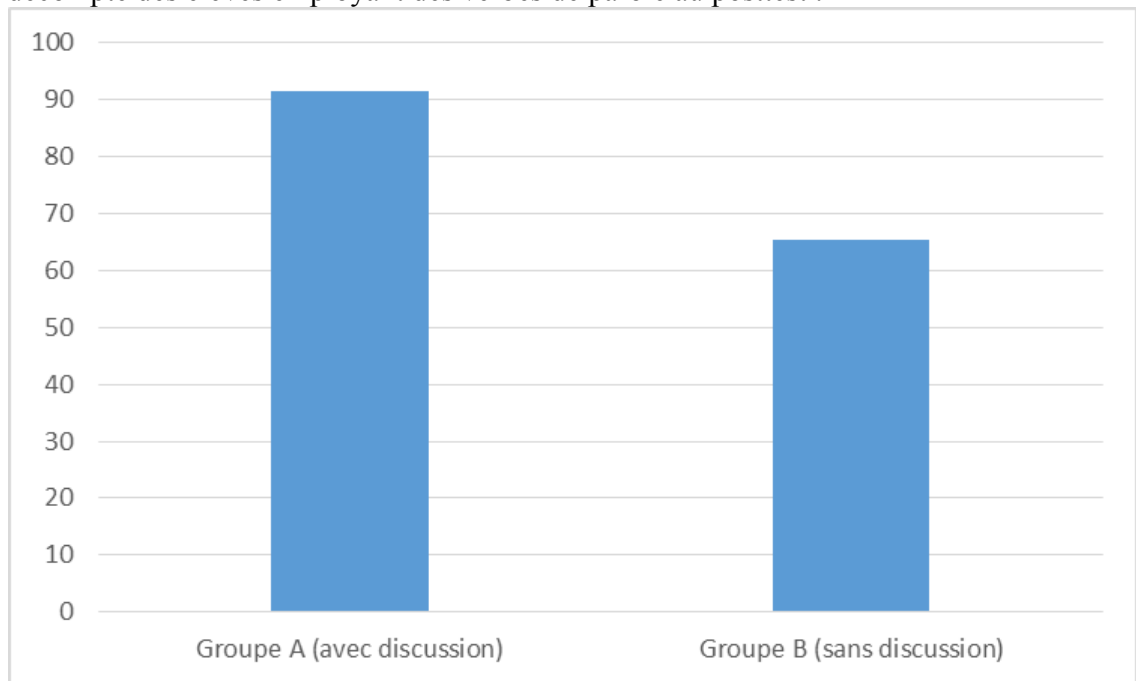

Fig. 2. Au posttest : pourcentages d'élèves utilisant des verbes de parole $(n=88)$

Cette première analyse des données issues du posttest nous apporte des éléments relatifs aux effets des séances ciblant des unités lexicales précises en didactique du lexique. En effet, nous constatons que l'ensemble des élèves, tous groupes confondus, progressent dans l'utilisation des verbes de parole. Par exemple, beaucoup de ceux qui n'en avait pas employé au prétest en emploient en posttest, mais ceux-là semblent suremployer à nouveau le verbe dire, bien que le verbe répondre, également très familier aux élèves, soit assez utilisé dans le posttest. Or, les élèves qui emploient dire ou répondre en posttest ne se réfèrent pas aux verbes étudiés. Nous pensons que, du fait qu'ils ont travaillé autour de six verbes de parole, ils mobilisent d'autres verbes qui, sans doute, leur sont plus familiers. Ainsi le fait de sélectionner des unités lexicales précises en didactique du lexique répond à l'objectif de structuration du lexique des élèves. Il leur permet en effet de mobiliser d'autres unités, ce qui a élargi leurs emplois des verbes de parole au posttest. Pour autant, il nous semble que le critère de sélection de ces unités n'est pas toujours la familiarité comme nous allons le voir ci-dessous en procédant à une analyse détaillée des données.

Une étude plus approfondie des données issues du posttest nous permet alors d'analyser cette variété d'emploi des verbes de parole. Comme précédemment, nous recensons les occurrences des verbes utilisés par les élèves dans leur dernière production :

Tableau 4. Au posttest : variété de verbes de parole utilisés en nombre d'occurrences $(n=88)$

\begin{tabular}{|l|l|l|}
\hline $\begin{array}{l}\text { Variété des verbes de } \\
\text { parole utilisés }\end{array}$ & $\begin{array}{l}\text { Groupe A } \\
\text { (avec discussion) }\end{array}$ & $\begin{array}{l}\text { Groupe B } \\
\text { (sans discussion) }\end{array}$ \\
\hline
\end{tabular}




\begin{tabular}{|l|r|r|}
\hline Hurler & 29 & 9 \\
\hline Murmurer & 19 & 2 \\
\hline Marmonner & 18 & 6 \\
\hline Conclure & 19 & 3 \\
\hline Déclarer & 33 & 4 \\
\hline Répliquer & 49 & 3 \\
\hline Répondre & 25 & 12 \\
\hline Demander & 10 & 4 \\
\hline S'exclamer & 6 & 3 \\
\hline Chuchoter & 3 & 1 \\
\hline Renchérir & 1 & 0 \\
\hline Dire & 134 & 65 \\
\hline Total & $\mathbf{3 4 6}$ & $\mathbf{1 1 2}$ \\
\hline
\end{tabular}

Ce tableau nous renseigne sur les emplois effectués par l'ensemble des élèves. Il nous amène à effectuer plusieurs constats.

Si nous le comparons au tableau décomptant les occurrences des verbes de parole utilisés au prétest, nous remarquons que la variété des verbes employés s'est accrue dans les deux groupes. Ainsi, non seulement les élèves sont plus nombreux à utiliser des verbes de parole, mais ces verbes sont également plus variés. Cependant, il apparait que les verbes mobilisés par les élèves du groupe B sont plus fréquents que ceux mobilisés par les élèves du groupe $\mathrm{A}$ : répondre est alors majoritairement employé à côté de dire qui reste prévalent. Certains élèves mobilisent aussi des unités peu fréquentes qui ont pu émerger grâce au travail sur le lexique, telles renchérir ou s'exclamer que nous supposons moins familières que dire ou répondre. Dans le groupe $\mathrm{B}$, cette plus grande variété ne répond qu'en partie à l'objectif d'accroissement du lexique puisque les élèves ont mobilisé des verbes qu'ils connaissaient sans doute déjà bien.

En revanche, les élèves du groupe A mobilisent une plus grande variété de verbes de parole, notamment les verbes du corpus étudié. L'écart entre les deux groupes est ainsi beaucoup plus important pour ces verbes. La différence entre le nombre d'occurrences de ces verbes utilisés dans le groupe A ou dans le groupe B apparait significative ce qui nous fait dire qu'elle est due au fait que, en A, les interactions verbales (telles que définies précédemment comme discussions lexicales) ont favorisé les apprentissages lexicaux.

Les élèves utilisent aussi des verbes apparaissant dans les paraphrases explicatives proférées lors des discussions puis écrites dans les livrets tels chuchoter qui a été mobilisé pour expliquer murmurer, ou répondre qui a été utilisé pour expliquer que répliquer signifiait répondre à quelqu'un. Enfin l'interprétation du tableau 4 amène un dernier constat: pour les élèves du groupe $A$, nous notons également un meilleur équilibre entre les verbes exprimant une émotion du personnage (hurler, murmurer, marmonner) ou une étape du dialogue (conclure, déclarer, répliquer), alors que les verbes exprimant une émotion étaient peu représentés dans le prétest.

L'analyse de quelques extraits des productions d'une élève du groupe A nous permet de mieux comprendre les effets des discussions qui ont eu lieu dans le groupe A (nous retranscrivons les textes d'élève) :

Tableau 5. Au prétest : exemple de production d'élève 
Production de Céline au prétest

Kirito et son amie Asuna parte visiter la forête au coure de leur voyage ils rencontrent une fille qui se nomme Toyauna.

- Qui éte vous? Je vous est déjà vu dans se jeu.

Comment tu t'appelle.

- Non tu ne ma jamais vu car je vient que d'arriver. Et je m'appelle Kirito et toi tu t'appelle comment.

- Je m'appelle Touyauna et je suis gardienne de la forêt. Et ta copine elle s'appelle comment?

- Elle s'appelle Azuna et elle est muette.

C'est ma meilleurs amie. Tu c'est ou c'est ma maison.

- Tu ne peux pas rentrer chez toi car tu doit troué les deux fées comme Azuna car elle pourons te renvoiller chez toi.

Tableau 6. Au posttest : exemple de production d'élève

\section{Production de Céline au posttest}

Deux personnages attendent leur amie qu'ils devaient retrouver et qui ne vient pas au rendez-vous.

Imagine ce que peuvent se dire les deux personnages qui attendent en écrivant leur dialogue.

- Le film va bientôt commencer, mais attendons encore un peu de temps déclare Mélina.

- Je ne suis pas d'accord allo sua cinéma.

- D'accord. Elle nous a posé un lapin.

Ils rentrent dans la salle de cinéma.

- Nous allons nous mettre pré de la porte, si Marie rentre elle nous vera. J'en sui sur.

Le film a commencé Marie arrive en hurlant.

- JULES, MÉLINA je uis la vous ète ou.

Jules répond à Marie

- Nous sommes l'a chuchote mais n'hurle pas!

- J'ai compris mais sa me dis pas ou vous ète.

- Nous sommes pré de la porte.

- Je vous rejoind.

À la fin du film il son resorti tout les trois. Ils se sont parler.

- C'est quoi la reson pour la qu'elle tu arrives en retard?

- Je suis en retard car ma maman m'a oblijet a ranger la maison.

- On te pardonne. Dit Mélina.

Et les amis partent chez eux.

Dans sa première production, Céline n'emploie aucun verbe de parole dans son dialogue. Dans le début du texte, cette absence peut paraitre logique car les personnages se présentent eux-mêmes : «comment tu *t'appelle », « ... et toi tu *t'appelle comment ». En revanche, dans la suite du texte l'absence des verbes de parole ne peut être expliquée par le déroulement du dialogue, mais plutôt par le fait que l'élève ne pense pas à mobiliser ceux qu'elle connait déjà.

La deuxième production de Céline nous parait révélatrice à plusieurs titres. D'une part elle montre que l'élève pense alors à mobiliser des verbes déjà connus. C'est l'analyse de son premier livret qui nous permet cette interprétation : à la question «as-tu déjà entendu ce mot? »Céline répond par l'affirmative pour tous les verbes sauf déclarer. En outre, dans cette deuxième production, les verbes déjà connus ne sont pas employés par Céline pour introduire les paroles des personnages, mais pour rendre compte de leur état d'énervement: " Marie arrive en *hurlent », « Nous sommes *l'a chuchote mais *n'hurle pas ». 
Le verbe que Céline disait ne pas connaitre est en revanche utilisé en début de dialogue pour introduire la première réplique du personnage.

Il nous semble que ces différents emplois, s'ils sont à mettre en relation avec le degré de connaissance que l'élève dit avoir des verbes étudiés, sont également à analyser au regard des discussions lexicales. En effet, lors des échanges, les consensus sur le sens des verbes ont généralement été assez rapides excepté pour déclarer et répliquer qui étaient moins familiers aux élèves. Ce sont les échanges qui ont eu lieu en début de parcours à propos de déclarer et murmurer qui nous intéressent ici car nous pensons qu'ils peuvent être à l'origine des emplois proposés par Céline. Concernant déclarer, la discussion a eu lieu à partir des expressions semi figées déclarer forfait et déclarer la guerre. Les élèves en ont conclu que déclarer signifiait bien dire quelque chose, mais qu'il s'agissait aussi d'exprimer le début d'une action. Voici l'extrait de leur échange qui illustre cette réflexion :

Ma. : Déclarer ça veut dire qu'on va abandonner

P. : Pourquoi tu dis ça Marine?

Ma. : Ben quand on déclare forfait...

P. : Ah, c'est qu'on abandonne... Et si on dit qu'on déclare la guerre, est-ce qu'on abandonne ?

Ju. : Non, on fait la guerre

P. : Ça veut dire quoi ça déclarer la guerre?

$\mathrm{Ju}$. : Oui, c'est dire qu'on va le faire. C'est pour commencer la guerre

Ma. : C'est quand on dit qu'on va le faire

Xa. : Des fois c'est officiel quand on fait une annonce

Cla. : Oui, c'est dire des choses officiellement, comme une déclaration

...

Les échanges se sont poursuivis et finalement les élèves ont paraphrasé déclarer par dire - dire publiquement quelque chose - annoncer dans leur livret. Pour déclarer, le recours au sème caractérisant un énoncé officiel est ainsi mis en avant par les élèves qui se fondent également sur leur interprétation du substantif déclaration dont le prototype est pour eux la déclaration d'impôts comme ils l'ont remarqué par la suite. Il nous semble que le fait d'avoir discuté davantage sur ce verbe, d'en avoir négocié le sens, a permis à Céline de se l'approprier au point d'oser le réemployer en production écrite. Elle sélectionne ainsi déclarer au début de son texte, alors qu'elle disait ne pas connaitre cette unité lexicale en début de dispositif dans les activités proposées dans le premier livret. Les élèves du groupe B emploient d'ailleurs assez peu déclarer : nous n'en dénombrons que 4 occurrences dans ce groupe alors que nous en trouvons 33 dans le groupe A. Ces analyses croisées entre les productions, les livrets et les échanges nous font dire que le fait d'avoir ménagé des temps de discussion a favorisé les élèves du groupe A. Concernant murmurer, Céline va se saisir aussi des échanges et des notes prises à l'issue de ces échanges en utilisant les remarques basées sur la relation de synonymie et d'antonymie proposée par certains de ses pairs :

P. : Alors, pour murmurer nous avons plusieurs propositions. Vous le connaissiez tous, n'est-ce pas?

Al. 1 : Murmurer c'est quand on parle pas fort

Ma. 1 : Oui

P. : Oui, ça vous fait penser à quoi alors ce mot ?

Pa. 1 : Ça fait penser à la cantine parce que Martine elle dit qu'on fait trop de bruit.

P. : Mais Ali vient de nous dire que murmurer ça voulait dire ne pas parler fort...

Pe. 1 : Oui, ben c'est ça, à la cantine il faut pas crier alors il faut chuchoter

Al. 2 : Murmurer quoi... C'est comme chuchoter

Ma. 2 : Parce que c'est parler sans hurler quand on murmure

P. : Toi Marine tu l'opposes à hurler alors

Ma. : Ben oui parce que si tu hurles tu parles fort

Cla. 1 : C'est pour être discret

P. : Hurler?

Cla. : Non, murmurer c'est quand on chuchote, on est discret

P. Ah, et oui... Par rapport à hurler c'est presque l'inverse, on murmure plutôt quand il ne faut pas faire trop de bruit ou quand on veut être discret comme dit Clara.

Bon alors, qu'est-ce qu'on décide d'écrire pour murmurer dans les deuxièmes livrets ? 
Pour murmurer, Céline s'empare des paraphrases explicatives et sélectionne chuchoter dans la réplique d'un personnage qui demande à un autre de chuchoter et non de hurler. Ces verbes n'introduisent pas alors des paroles de personnages, mais ils apparaissent dans une réplique réclamant la discrétion. Ces types d'emploi sont des réussites car ils montrent que Céline s'est suffisamment approprié les unités lexicales pour les utiliser autrement que comme des verbes introducteurs de paroles. Elle devait déjà assez bien connaitre chuchoter (sélectionné par préférence à murmurer, sans doute moins familier) et hurler, mais les interactions ont pu favoriser ces types d'emplois. Ici aussi, les discussions, basées sur différentes structures du système lexical, semblent être favorables au réemploi.

Ces résultats sont confirmés par le décompte du nombre d'élèves utilisant les verbes de parole étudiés :

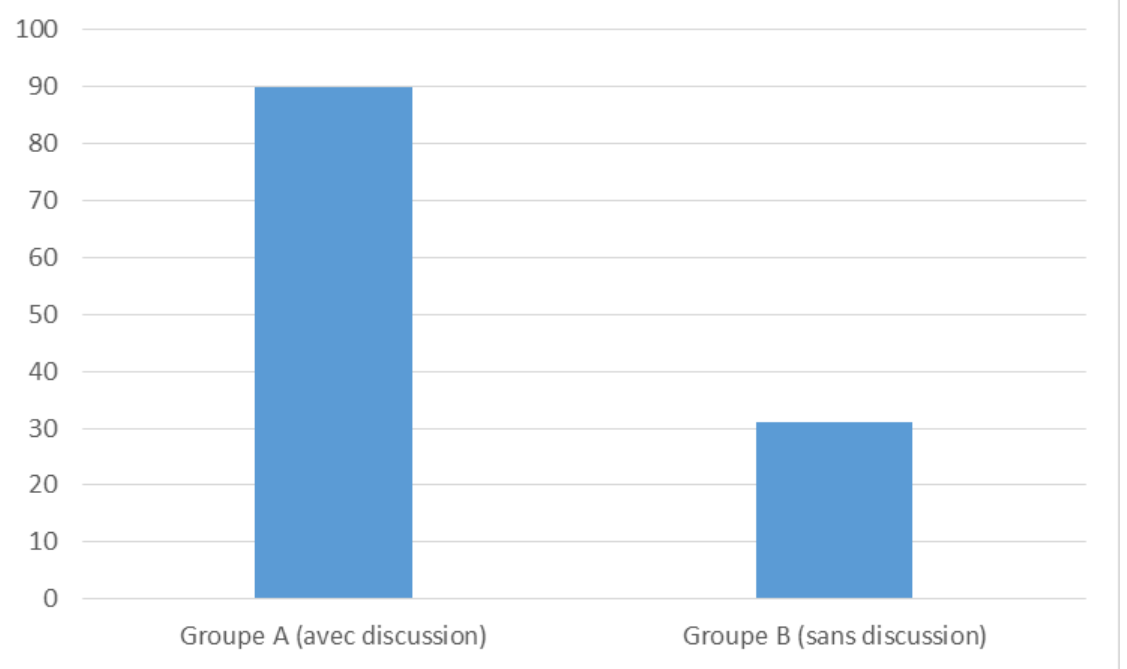

Fig. 3. Au posttest : pourcentages d'élèves utilisant des verbes de parole étudiés $(\mathrm{n}=88)$

Remarquons que cette figure concerne le nombre d'élèves employant les verbes de parole étudiés et non le nombre d'élèves utilisant tout verbe de parole comme le présente la figure 2. Ici aussi l'écart entre les deux groupes s'est avéré significatif. Cette figure 3 confirme alors que les élèves ayant bénéficié des discussions entrainant la coconstruction du sens des lexies étudiées ont développé de meilleures performances dans le réemploi des verbes étudiés. Ils sont en effet beaucoup plus nombreux à avoir proposé une grande variété de verbes de parole comme le suggérait le tableau 3 dénombrant les occurrences utilisées par les élèves.

Ainsi, tous les élèves progressent dans l'utilisation des verbes de parole, mais ceux du groupe B utilisent des verbes moins variés que ceux du groupe A qui utilisent davantage (significativement plus) les verbes étudiés ainsi que les verbes utilisés dans les paraphrases explicatives. Les discussions lexicales ont ainsi un impact positif sur les capacités de réemploi à moyen terme et elles favorisent la variété d'emploi des verbes de parole.

À ce stade de l'analyse nous pouvons donc dire qu'en fin de parcours il semble que :

$\checkmark \quad$ Le fait d'opérer une sélection des unités lexicales à étudier invite les apprenants à mobiliser d'autres unités reliées notamment par des liens sémantiques aux unités ciblées par l'enseignement ; ces autres unités peuvent leur être plus familières, mais ils n'y avaient pour autant pas eu accès avant l'activité.

$\checkmark \quad$ Le fait de prévoir et organiser des temps de discussion durant les séances consacrées au lexique favorise les apprentissages lexicaux, ces temps efficients sont ainsi à intégrer en didactique du lexique. 
Ainsi, « comme le dit J.F. Halté (1995), on peut « souhaiter qu'on enseigne [...] en exploitant les moments de négociation du sens » dont on pose qu'ils favorisent l'élaboration d'objets de connaissance ou transforme la connaissance de ces objets » (Tauveron, 2001, p. 215).

\section{Conclusion}

Les discussions lexicales telles que nous les avons définies: présentant une part argumentative importante, s'appuyant sur les structures du lexique et proposées à des moments opportuns dans le dispositif d'enseignement paraissent donc favorables au développement de la compétence lexicale. Les élèves qui en ont bénéficié réemploient les verbes étudiés et proposent en outre une variété dans l'utilisation des verbes de parole. Il nous semble que ces élèves, en discutant de leur appréhension des unités qui leur étaient proposées, en ont accru leur compréhension. Le fait de prévoir et organiser des temps de discussion dans les séances d'étude du lexique les a engagés dans la réflexion lexicale. Dans une didactique du lexique qui s'organise autour des structures sémantiques, syntagmatiques et morphologiques du système lexical, intégrer judicieusement ces discussions serait ainsi moyen supplémentaire de favoriser les apprentissages lexicaux. Pour conforter ces premiers résultats il s'agit maintenant de tester les élèves au bout de plusieurs mois afin d'évaluer les effets des apprentissages lexicaux à plus long terme. Par ailleurs, l'empan temporel de la recherche ici présentée reste relativement réduit ; sans doute le déploiement d'un dispositif sur l'ensemble d'une année scolaire rendrait-il mieux compte des effets possibles des discussions lexicales sur les apprentissages lexicaux.

\section{Références}

Aurnague, M., \& Garcia-Debanc, C. (2016). Les verbes de déplacement comme contenu d'enseignement du lexique à l'école primaire : modélisation sémantique et analyse de productions d'élèves. Actes du 5e Congrès Mondial de Linguistique Française. doi: https://doi.org/10.1051/shsconf/20162707001

Chabanne, J.-C., \& Bucheton, D. (2002). Parler et écrire pour penser, apprendre et se construire. L'écrit et l'oral réflexifs. Paris: PUF.

Chanfrault-Duchet, M.-F. (2004). Vers une approche syntagmatique du lexique en didactique du français. Dans É. Calaque, \& J. David, Didactique du lexique : contextes, démarches, supports (p. 103 à 114). Bruxelles: De Boeck.

Jaubert, M., \& Rebière, M. (2002). Parler et débattre pour apprendre : comment caractériser un "oral réflexif" ? Dans J.-C. Chabanne, \& D. Bucheton, Parler et écrire pour penser, apprendre et se construire. L'écrit et l'oral réflexifs (p. 163 à 186). Paris: PUF.

Kerbrat-Orecchioni, C. ([1990] 1998). Les interactions verbales. Approche interactionnelle et structure des conversations. Paris: Armand Colin.

Laferrière, T. (2005). Les communautés d'apprenants en réseau au bénéfice de l'éducation. Encounters on Education 6, p. 5 à 21.

Lamiroy, B., \& Charolles, M. (2008). Les verbes de parole et la question de l'(in)transitivité. Discours [En ligne], 2. Consulté le novembre 7, 2017, sur http://discours.revues.org/3232 ; DOI : $10.4000 /$ discours. 3232

Sardier, A. (2015). Construire la compétence lexicale : quelle place en didactique pour le cotexte ? Thèse de doctorat, Grenoble: UGA. Consulté le octobre 25, 2017, sur https://tel.archivesouvertes.fr/tel-01424599/document

Sardier, A. (2016). Enseignement-apprentissage du lexique : vers le réemploi lexical en classe de grande section de maternelle. Repères 54, p. 207 à 232.

Sardier, A., \& Grossmann, F. (2010). Comment favoriser le réemploi lexical ? Recherches $n^{\circ} 53$ Lexique Vocabulaire, p. 9 à 29.

Tauveron, C. (2001). Modes de gestion de l'interaction : effets sur les postures conversationnelles des élèves et les apprentissages disciplinaires. Dans M. Grandaty, \& G. Turco, L'oral dans la classe. Discours, métadiscours, interactions verbales et construction de savoirs à l'école primaire (p. 185 à 221). Paris: INRP. 
Tremblau, O., \& Polguère, A. (2014, juillet). Une ontologie linguistique au service de la didactique du lexique. Dans F. Neveu, P. Blumenthal, L. Hriba, G. Gerstenberg, J. Meinschaeffer, \& S. Prévost, 4 e Congrès Mondial de Linguistique Française (CMLF) (p. 1173 à 1188). Berlin: EDP Sciences.

Triquenot, A. (2011). L'analyse du procès : un exemple d'articulation entre sémantique et syntaxe. Syntaxe et sémantique 12, p. 165 à 188. Consulté le novembre 13, 2017, sur

https://www.cairn.info/revue-syntaxe-et-semantique-2011-1-page-165.htm 


\section{Annexe 1 : extraits du premier livret des élèves}

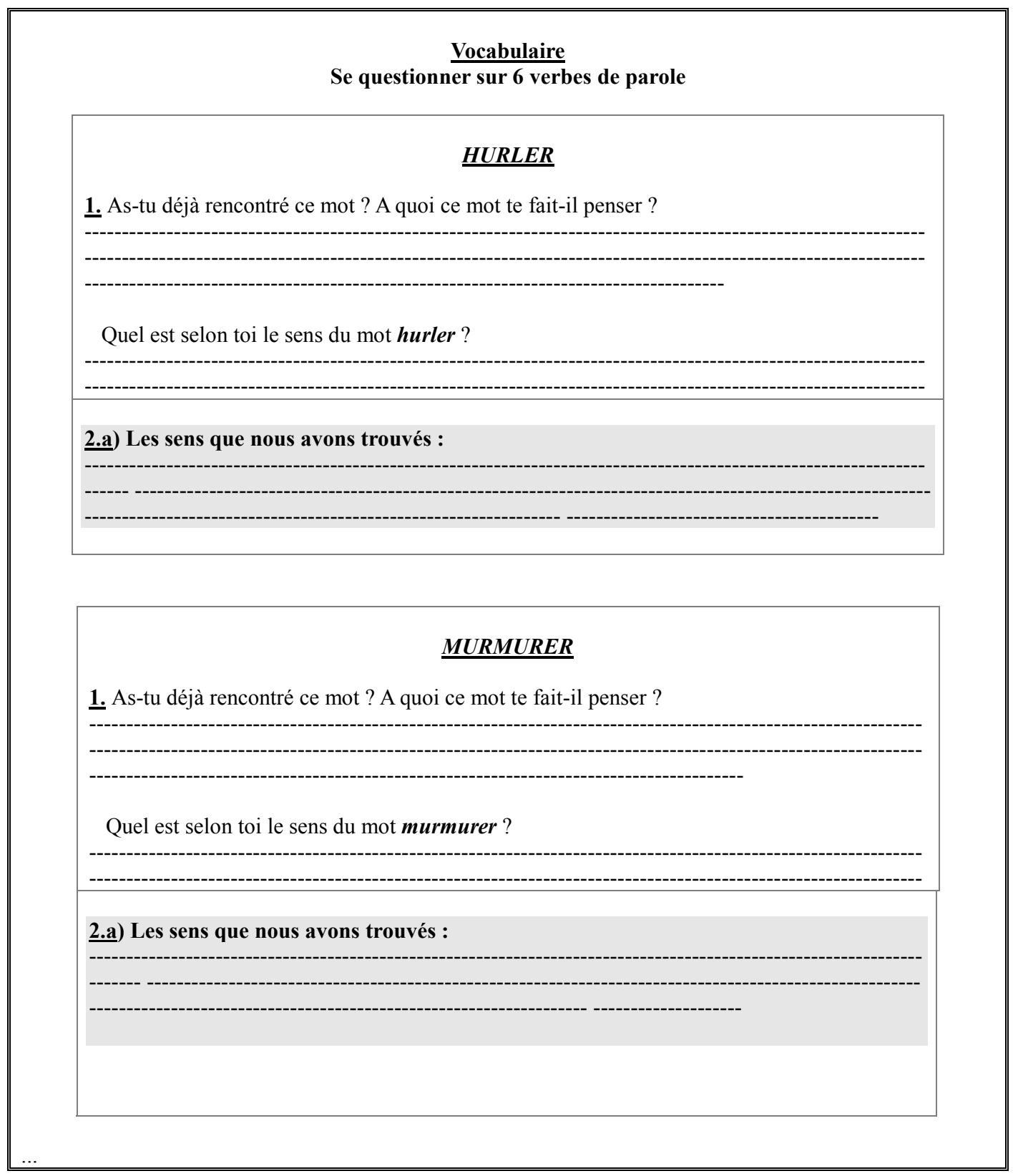




\section{Annexe 2 : extraits du deuxième livret des élèves}

\section{Connaitre et utiliser 6 verbes de parole}

Hurler Murmurer Marmonner

Conclure Déclarer Répliquer

\section{HURLER}

$\underline{\text { 2.b) }}$ Je rencontre hurler dans des phrases et j'en déduis son sens :

- « Dès le coucher du soleil, plusieurs chiens hurlent longuement à la mort... » (Bloy, Journal, 1907, p. 356).

Sens de hurler :

- « Comme personne ne l'écoutait, elle hurla ce qu'elle avait à dire» Sens de hurler :

- «Face au loup qui arrivait vers lui, il hurla de peur"

Sens de hurler :

\section{Bilan : les sens de hurler :}

4. Pour mieux comprendre et employer hurler : - je regarde le contexte :

- les personnages (humains ou non)

- les émotions des personnages (en colère, content, timide...)

- je regarde les mots qui sont autour de hurler : les noms et les adverbes

- hurler de peur

- hurler de colère

- hurler $\underline{\underline{\text { longuement }}} \underline{\underline{a ̀ ~ l a ~ m o r t}}$

5. Un mot de la même famille qu'hurler : 


\section{MURMURER}

2.b) Je rencontre murmurer dans des phrases et j'en déduis son sens :

- «Marie murmura tout doucement qu'elle avait fait une bêtise, mais elle n'avait pas envie qu'on l'entende... »

Sens de murmurer :

- « En marchant sur le sentier, il entendit la cascade murmurer au fond de la vallée » Sens de murmurer :

\section{Bilan : les sens de murmurer :}

\section{Pour mieux comprendre et employer murmurer :}

- je regarde le contexte :

- les personnages (humains ou non)

- les émotions des personnages (en colère, content, timide...)

- je regarde les mots qui sont autour de murmurer: les noms et les adverbes

- la cascade murmure

- murmurer tout doucement

5. Un mot de la même famille que murmurer :

\footnotetext{
${ }^{\mathrm{i}}$ Sur le concept de réflexivité en didactique, voir notamment Chabanne \& Bucheton, (dir.), 2002

${ }^{i i}$ Nous menons cette étude intitulée Écoles Éloignées en Réseau. Étude des effets d'un travail en réseau sur les apprentissages lexicaux d'élèves de cycle 3 au sein de l'université et en étroite collaboration avec
} 
le rectorat.

iii Pour une analyse des effets de la prise en compte du cotexte, et notamment de sa structuration grammaticale sur le développement de la compétence lexicale, voir Sardier, 2015.

iv Les devinettes lexicales s'appuient sur les différentes structures du système. Ainsi les élèves ont-ils proposé des devinettes telles « je commence par un $m$, je signifie parler doucement et je peux être le contraire de hurler, qui suis-je ? », ou bien « je contiens un $n$ et je termine la discussion, qui suis-je ?», etc. 